\title{
BEAM TRANSFER FUNCTION AND LANDAU DAMPING
}

\begin{abstract}
BNL $=-42939$
J.M. Wang

DE89 015111

May 1989

Presented at the 1988 Joint US-CERN Particle Accelerator School, Capri, Italy October 19-25, 1988
\end{abstract}

NATIONAL SYNCHROTRON LIGHT SOURCE

BROOKHAVEN NATIONAL LABORATORY ASSOCIATED UNIVERSITIES, INC. UPTON, LONG ISLAND, NEW YORK 11973

Under Contract No. DE-AC02-76CH00016 with the UNITED STATES DEPARTMENT OF ENERGY 


\section{Table of Contents}

CHAPTER

PAGE

1. Introduction 1

2. Beam Polarization 2

3. Beam Transfer Function 3

4. Weak Landau Damping Limit 6

5. Landau Damping and Resonant Energy Exchange 9

$\begin{array}{ll}\text { ó. References } & 18\end{array}$

\section{DISCLAIMER}

This repont was prepared as an account of work sponsored by an agency of the Urited States Government. Neither the United States Government nor any agency thereof, nor any of their employees, makes any warranty, express or implied, or assumes any legal liability or responsibility for the accuracy, completeness, or usefulness of any information, apparatus, product, or process disclosed, or represents that its use would not infringe privately owned rights. Reference herein to any specific commercial product, process, or service by trade name, trademark, manufacturer, or otherwise does not necessarily constitute or imply its endorsement, recommendation, or favoring by the United States Government or any agency thereof. The views and opinions of authors expressed herein do not necessarily state or rellect those of the United States Government or any agency thereof. 


\author{
Jiunn-Ming Wang \\ National Synchrotron Light Source \\ Brookhaven National Laboratory \\ Upton, NY 11973
}

\title{
1. INTRODUCTION
}

This note consists of two parts. In the first part we study the initial value problem of the longitudinal motion of a particle in a coasting beam. The result is expressed in terms of a beam transfer function (BTF) [1-5] which relates the beam polarization to the external longitudinal electric field and the initial condition. In the absence of the beam-induced electric field, the beam transfer function $F$ is the sum of the free particle Green's functions of the beam particles. If the beam-induced longitudinal electric field is taken into account, we obtain a feedback loop, and the BTF is modified to $G=F / D$ where the dielectric function $D$ describes the collective behavior of the beam particles. The condition $D=0$, called the dispersion relation, relates the coherent frequency of the coherent state to the wave number. The treatment of this entire note is within the framework of a linear approximation. We adopt a method [6] introduced in 1985 to describe the longitudinal perturbation of the beam particle position.

In 1946, Landau [1] demonstrated that the frequency spread tends to damp the coherent longitudinal oscillation of a stationary plasma. The idea of collisionless damping within the linear approximation remained a puzzle until 1961 when Dawson [7] presented an intuitive explanation of Landau damping. His argument is based on energy absorption by resonant beam particles. He divided the beam particles into two groups:

1. Resonant particles: that small number of particles whose oscillation frequencies are the same as, or very close to, the frequency of the plasma wave.

2. Main plasma: the rest of the particles.

Dawson postulated that the main plasma is the carrier of the plasma wave. The resonant particles stay in phase and thus exchange energy strongly with the wave. For a plasma with a single-hump velocity distribution such as a Maxwellian plasma, the resonant particles on the average gain energy and thus diminish the strength of plasma oscillation. He also demonstrated that his argument leads to a damping rate identical to that calculated by Landau. In 1965, Hereward [8] explained the Landau damping of the transverse coherent instability in storage rings with the same resonant energy absorption idea.

In the second part of this note, the work of Landau and Dawson on longitudinal plasma oscillation of a stationary plasma is adapted to the longitudinal coherent wave of a coasting beam. In order to be mathematically precise, we consider only a very specific case: that of a space-charge wave below the transition energy.

There is a complication in treating the Landau damping of the longitudinal coherent wave in a storage ring: the beam drifts around the ring with angular velocity $\omega_{0}$. As a consequence, for a fast space-charge wave (the wave with angular phase velocity greater than $\omega_{0}$ ) the resonant particles absorb energy from the wave, but for a slow wave they give 
energy to the wave; and yet both fast and slow waves are Landau damped. This can be understood from the fact that the fast wave is a positive energy wave and the slow wave is a negative energy wave in the sense of the kinetic power theorem (or small signal power theorem) [9-11]. In a negative energy wave, the wave-carrier particles on the average drift with less kinetic energy than they would without the wave excitation. Thus, by giving energy to the slow wave, the resonant particles are damping the wave out.

In Section 4 the Landau damping rate is calculated in a weak damping limit by solving a dispersion relation. Also, the difference between the Landau damping and the damping from decoherence (phase mixing) is emphasized. In Section 5, the same damping rate is calculated by using the kinetic power theorem. In the Appendix, we review the well-known properties of the longitudinal space-charge waves in a storage ring [12, 13].

\section{BEAM POLARIZATION}

We investigate in this section how the longitudinal displacements of the beam particles from their nominal positions induce the line-charge density and the local current. The treatment is within a linear approximation.

Let us denote the nominal azimuthal position of the $j-$ th particle, $j=1,2, \cdots, N$, by

$$
\theta_{\mathrm{j}}=\psi_{\mathrm{j}}+\omega_{0}\left(1-\eta \varepsilon_{\mathrm{j}}\right) t
$$

where $\psi_{\mathrm{j}}$ is the initial position $\varepsilon_{\mathrm{j}} \doteq\left(\mathrm{p}_{\mathrm{j} 2}-\mathrm{p}_{0}\right)^{\prime} \mathrm{p}_{0}$ is the fractional momentum deviation of the particle, and $\eta=1 / \gamma_{t}-1 / \gamma_{2}$, and define the perturbation $\alpha$ of the particle position implicitly by

$$
\theta_{j}=\psi_{j}+\omega_{0}\left(1-\eta \varepsilon_{j}\right) t+\alpha\left(\theta_{j}, \varepsilon_{j}, t\right)
$$

Within linear approximation, the last equation becomes

$$
\theta_{j}=\psi_{j}+\omega_{0}\left(1-\eta \varepsilon_{j}\right) t+\alpha\left[\psi_{j}+\omega_{0}\left(1-\eta \varepsilon_{j}\right) t, \varepsilon_{j}, t\right] .
$$

The line-charge density and the local current are, respectively,

$$
\rho(\theta, t)=\frac{e}{R} \sum_{j=1}^{N} \delta\left[\theta-\theta_{j}(t)\right]
$$

and

$$
I(\theta, t)=e \sum_{j=1}^{N} \delta\left[\theta-\theta_{j}(t)\right] \frac{d}{d t} \theta_{j}(t)
$$

where $R$ is the ring radius.

If we assume $\psi_{j}$ and $\varepsilon_{j}$ to be uncorrelated, and denote by $g(\varepsilon)$ the distribution function in $\varepsilon$, with $\int \mathrm{d} \varepsilon g(\varepsilon)^{3}=1$, then simple calculations lead to

$$
\rho(\theta, t)=\frac{e N}{2 \pi R}\left[1-\int \operatorname{d\varepsilon g}(\varepsilon) \alpha^{\prime}(\theta, \varepsilon, t)\right]
$$


and

$$
I(\theta, t)=\frac{e N}{2 \pi}\left[\omega_{0}(1-\eta\langle\varepsilon\rangle)+\int \operatorname{d} \varepsilon g(\varepsilon) \dot{\alpha}(\theta, \varepsilon, t)\right]
$$

where $\langle\varepsilon\rangle=\int d \varepsilon \varepsilon g(\varepsilon), \alpha^{\prime}=\partial \alpha / \partial \theta, \alpha=\partial \alpha / \partial t$. Because of Eqs. (2.6) and (2.7), we shall call

$$
\alpha(\theta, t)=\int d \varepsilon g(\varepsilon) \alpha(\theta, \varepsilon, t)
$$

the longitudinal beam polarization.

\section{BEAM TRANSFER FUNCTION}

We solve in this section the initial value problem of the longitudinal beam particle motion in an external longitudinal electric field $E^{\text {ext }}$, and thereby introdace the concepts of the beam transfer function (BTF) and the dielectric function Di $\omega$ ). A convenient method of solving a linear initial value problem is the Laplace-Fourier transform (LFT), and the BTF is the LFT of the Green's function of the equation of motion. The dielectric function will be introduced by considering the effects of the electric field $E^{\text {ind }}$ induced by the perturbed beam itself. The total electric field is

$$
E(\theta, t)=E^{e x t}(\theta, t)+E^{i n d}(\theta, t) .
$$

Let us first review some results of the Laplace-Fourier transform. The LFT $\tilde{\mathrm{f}}(\omega)$ of a function $f(t)$ is defined by

The inverse of this transformation is

$$
\bar{f}(\omega)=\frac{1}{2 \pi} \int_{0}^{\infty} d t f(t) e^{i \omega t} .
$$

$$
f(t)=\int_{a-i \infty}^{a+i \infty} d \omega \tilde{f}(\omega) e^{-i \omega t},
$$

where " $a$ " is chosen such that $\bar{f}(\omega)$ is analytic in the upper half of the complex $\omega$-plane defined by Imaginary $(\omega) \geq a$. Note that $f(t)$ as defined by the last integral vanishes for $t<0$. The LFT of $\mathrm{f}(t)$ can easily be obtained by a partial integration:

$$
f(\omega)=\frac{1}{2 \pi} \int_{0}^{\infty} d t \hat{f}(t) e^{i \omega t}=-\frac{1}{2 \pi} f(0)-i \omega \tilde{f}(\omega)
$$




\section{A. Equation of Motion and BTF}

The equation of motion of the beam particle in the total electric field $E(\theta, t)$ is

$$
\frac{d^{2}}{d t^{2}} \alpha(\theta, \varepsilon, t)=-e \bar{\eta} E(\theta, t)
$$

with

$$
\frac{d}{d t}=\frac{\partial}{\partial t}+\omega_{0}(1-\eta \varepsilon) \frac{\partial}{\partial \theta}
$$

and $\bar{\eta}=\eta \omega_{0} / p_{0}$. It is convenient to Fourier decompose (3.2) in the variable $\theta$. We define the Fourier components $f_{n}$ of any function $f(\theta)$ by $f(\theta)=\sum f_{n} \exp ($ in $\theta)$. The equation of motion becomes

$$
\left[\frac{\partial}{\partial t}+i n \omega_{0}(1-\eta \varepsilon)\right]^{2} \alpha_{n}(\varepsilon, t)=-\operatorname{en} E_{n}(t)
$$

Let us introduce a new variable $\Omega=-n \omega_{0} \eta \varepsilon$, and write $\alpha(\Omega, t) \equiv \alpha(\varepsilon, \mathfrak{t})$. Then a Laplace-Fourier transformation of (3.4) leads to

$$
\tilde{\alpha}_{n}(\Omega, \omega)=e \bar{\eta}\left[\frac{E_{n}^{\text {ind }}(\omega)+E_{n}^{\text {ext }}(\omega)}{\left(\omega-n \omega_{0}-\Omega\right)^{2}+\bar{A}_{n}(\Omega, \omega)}\right]
$$

where $\tilde{A}_{n}$ describes the initial beam polarization:

$$
\bar{A}_{n}(\Omega, \omega)=-\frac{1}{2 \pi e \bar{\eta}} \frac{\left\{\dot{\alpha}_{n}(\Omega, 0)-i \omega \alpha_{n}(\Omega, 0)\right\}}{\left(\omega-n \omega_{0}-\Omega\right)^{2} .}
$$

These two equations would have been the solution to the equation of motion had $E^{\text {ind }}$ been negligible. To include the effects of the beam-particle self-interaction on the solution, we spell out below the dependence of $E^{\text {ind }}$ on $\alpha$.

From now on, the discussions will be confined to a specific Fourier component $n, n>$ 0 ; therefore, the subscript $n$ will be omitted from our notation.

Recalling the definition of the impedance function $Z(\omega)$, and the relationship (2.7) between the local current $I(\theta, t)$ and $\alpha(\theta, t)$, we have

$$
\overline{\mathrm{E}}^{\mathrm{ind}}(\omega)=-\frac{1}{2 \pi \mathrm{R}} \bar{I}(\omega) Z(\omega)=\mathrm{i} \frac{\mathrm{eN}}{4 \pi^{2} \mathrm{R}} \omega \bar{\alpha}(\omega) Z(\omega),
$$

where

$$
\tilde{\alpha}(\omega)=\int \mathrm{d} \Omega \mathrm{h}(\Omega) \tilde{\alpha}(\Omega, \omega)
$$

with the distribution function in $\Omega$ defined by $h(\Omega)=g(\varepsilon) d \varepsilon / d \Omega$.

It is convenient to introduce the scaled impedance functions by 


$$
V(\omega)+i U(\omega)=\left(e^{2} N \bar{\eta} / 4 \pi^{2} R\right) \omega Z(\omega)
$$

where $V$ corresponds to the resistive part and $U$ to the reactive par. $V$ and $U$ are in units of (frequency) ${ }^{2}$.

If we eliminate $E^{\text {ind }}$ in (3.5) by using (3.7), and then operate with $\int \mathrm{d} \Omega \mathrm{h}(\Omega)$ on the resulting equation, we obtain the result we have been seeking; namely, the relationship between $\tilde{\alpha}(\omega)$ and the input (external field and the initial condition). The result can best be described in terms of a block diagram with one feedback loop, as given in Fig. 1.

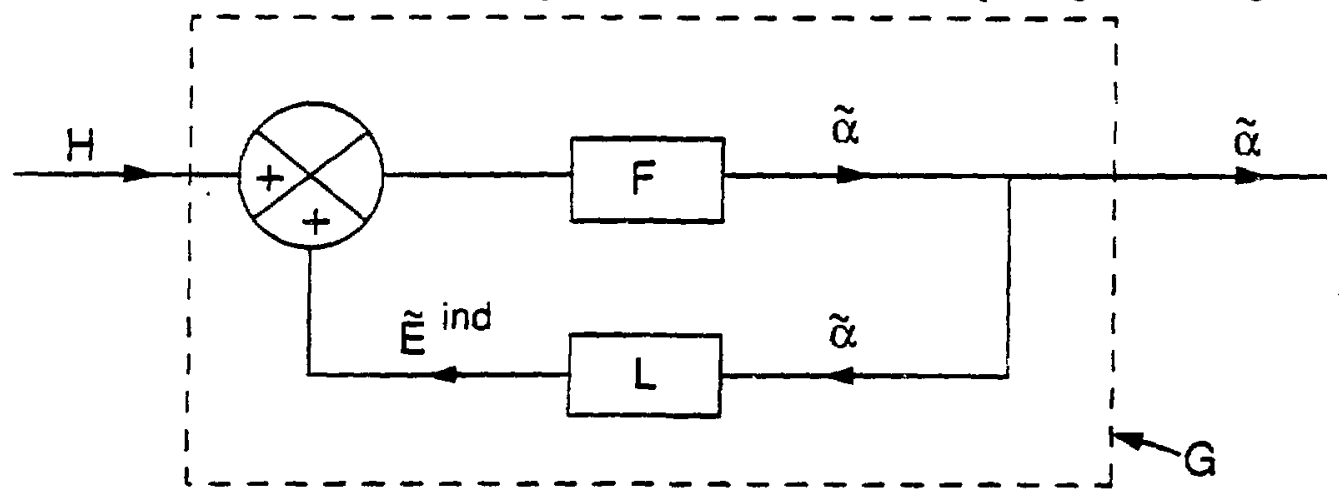

Fig. 1. Block diagram for the beam transfer function $G(\omega)$.

$$
\begin{aligned}
& \tilde{\alpha}(\omega)=G(\omega) H(\omega) ; \\
& \Lambda(\omega)=\int d \Omega h(\Omega) /\left(\omega-n \omega_{0}-\Omega\right)^{2} ; \\
& G=F / D ; \quad F=e \bar{\eta} \Lambda ; L=-(U-i V) / e \bar{\eta} ; \\
& D=1-L F=1+(U-i V) \Lambda ; \\
& H=E^{e x t}+\bar{A} / \Lambda .
\end{aligned}
$$

$\mathrm{G}(\omega)$ is the beam transter function, $H(\omega)$ is the input, we shail call $D(\omega)$ the longitudinal dielectric function. Note that the input $H(\omega)$ is a combination of $E^{\text {ext }}$ and the initial polarization.

$\alpha(t)$ is the inverse LFT of $\tilde{\alpha}(\omega)$,

$$
\alpha(t)=\int d \omega \frac{F(\omega)}{D(\omega)} H(\omega) e^{-i \omega t}
$$

where the integrand depends on $\Lambda(\omega)$ through the relations above. As explained in the beginning of this section, the $\omega$-contour in (3.10) should lie above all singularities of the integrand. This in rum implies that in the integral representation for $\Lambda(\omega)$ above, the $\Omega$ contour should lie under the singularity at $\Omega=\omega-n \omega_{0}$. It is also imporant to note thar a simple zero of $\mathrm{D}(\omega)$ yields a pole in the integrand of (3.10). 


\section{Dielectric Function}

To find the physical meaning of $D(\omega)$, let us assume for simplicity that the initial beam polarization vanishes, $\tilde{A}(\omega)=0$. Then, from the block diagram,

$$
\mathrm{E}^{\text {ind }}=\mathrm{L} \tilde{\alpha}=L G \mathrm{E}^{\mathrm{ext}}=\mathrm{L} \frac{\mathrm{F}}{\mathrm{D}} \tilde{\mathrm{E}}^{\mathrm{ext}} .
$$

Therefore, the total electric field

$$
E=E^{\text {ind }}+E^{\text {ext }}=\left(1+L \frac{F}{D}\right) E^{\text {ext }} \text {. }
$$

But, since $D+L F=1$, we obtain finally

$$
E(\omega)=E^{e x l}(\omega) / D(\omega) .
$$

This expression explains why $D(\omega)$ is called the dielectric function.

\section{C. Coherent State and Dispersion Relation}

The coherent frequency $\omega_{C}$ of a coherent state is related to the wave number $\mathrm{n}$ by the dispersion relation

$$
D\left(\omega_{C}\right)=0
$$

or

$$
1+(U-i V) \int d \Omega \frac{h(\Omega)}{\left(\omega_{C}-n \omega_{0}-\Omega\right)^{2}}=0 .
$$

Let us define the coherent frequency shift $\Omega_{C}=\omega_{C}-n \omega_{0} \equiv \Omega_{R}+i \Omega_{I}$. Suppose that the integrand of (3.10) has a simple pole at $\omega=n \omega_{0}+\Omega_{C}$ and that it is analytic in the upper half $\omega$-plane defined by Imaginary $(\omega)>\Omega_{I}$, then

$$
\alpha(\mathrm{t})-\mathrm{e}^{\Omega_{\mathrm{I}} \mathrm{t}} \text { as } t \rightarrow \infty \text {. }
$$

The coherent wave is therefore unstable if $\Omega_{1}>0$, it is damped if $\Omega_{1}<0$, and the coherent oscillation is stationary if $\Omega_{1}=0$. It should be emphasized that an expression like (3.13) is an asymptotic expansion; it is meaningful only in the limit $t \rightarrow \infty$.

\section{WEAK LANDAU DAMPING LIMIT}

We solve the dispersion relation in a weak damping limit when the Landau damping coefficient $-\Omega_{l}$ is small, and calculate the damping coefficient. The model we solve is defined as follows:

(i) The source of impedance is the space-charge force, and the beam is below the transition energy $(\eta<0)$. This implies $V=0, U<0$.

(ii) The beam cross section is much smaller than the beam-chamber cross section, also the beam density is constant across the beam so that the electric field is constant across the beam.

(iii) $\mathrm{E}^{\mathrm{ext}}=0$. 
The dispersion relation is then, from (i) above,

$$
1+U \cdot \int_{-\infty}^{\infty} d \Omega \frac{h(\Omega)}{\left(\Omega_{C}-\Omega\right)^{2}}=0 .
$$

\section{A. Cold Beam}

Let us first consider the simplest case where

Then, from (4.1),

$$
h(\Omega)=\delta(\Omega) .
$$

$$
\Omega_{C}= \pm \sqrt{-U} .
$$

Since $U$ is negative, the space-charge waves are stationary for a cold beam. The phase angular velocity of a coherent wave is $\omega_{C} / n=\omega_{0}+\Omega_{C} / n$; therefore, the plus sign in (4.3) corresponds to a fast wave and the negative sign, to a slow wave.

\section{B. Weak Damping Limit}

A warm beam is considered here. Let us add more provisos to the model.

(iv) $h(\Omega)$ is Gaussian; $h(\Omega)=(1 / \sqrt{2 \pi} \sigma) \exp \left(-\Omega^{2} / 2 \sigma^{2}\right)$.

(v) $-\sigma^{2} / U \ll 1$ : The coherent frequency lies on the tail of the beam frequency spectrum.

(vi) $\Omega_{\mathrm{I}} / \sigma<1$ : Weak damping.

The condition (vi) is to be verified by the resilt.

Integrating by parts, the dispersion relation can also be written as

$$
1-U \int d \Omega \frac{h^{\prime}(\Omega)}{\Omega_{C}-\Omega}=0 \text {. }
$$

Expanding the dispersion integral in power series in $\Omega_{I}$, and recalling that the $\Omega$-contour in the integral is under the pole at $\Omega=\Omega_{\mathrm{C}}$, we have

$$
\begin{aligned}
\int \mathrm{d} \Omega \frac{h^{\prime}(\Omega)}{\Omega_{\mathrm{C}}-\Omega} & =\sum_{n=0}^{\infty} \frac{\left(\mathrm{i} \Omega_{\mathrm{I}}\right)^{\mathrm{n}}}{\mathrm{n} !} \frac{\partial^{\mathrm{n}}}{\partial \Omega_{\mathrm{R}}^{\mathrm{n}}} \int \mathrm{d} \Omega \frac{\mathrm{h}^{\prime}(\Omega)}{\Omega_{\mathrm{R}}-\Omega} \\
& \equiv f \mathrm{~d} \Omega \frac{\mathrm{h}^{\prime}(\Omega)}{\Omega_{\mathrm{R}}-\Omega}+\mathrm{i}\left[\Omega_{\mathrm{I}} f \mathrm{~d} \Omega \frac{\mathrm{h}^{\prime \prime}(\Omega)}{\Omega_{\mathrm{R}}-\Omega}-\pi \mathrm{h}^{\prime}\left(\Omega_{\mathrm{R}}\right)\right] .
\end{aligned}
$$


where $f$ denotes the principal value of the integral. The dispersion relation now becomes

$$
\left\{\begin{array}{l}
1-U f d \Omega \frac{h^{\prime}(\Omega)}{\Omega_{R}-\Omega}=0, \\
\Omega_{I}=\pi h^{\prime}\left(\Omega_{R}\right) / f d \Omega \frac{h^{\prime \prime}(\Omega)}{\Omega_{R}-\Omega}
\end{array}\right.
$$

From (4.4) and the assumption (y).

$$
\Omega_{R} \equiv \pm \sqrt{-U}
$$

Equation (4.5) can also be written as

$$
\Omega_{\mathrm{I}}=\frac{\pi}{2} h^{\prime}\left(\Omega_{\mathrm{R}}\right) / f \mathrm{~d} \Omega \frac{\mathrm{h}(\Omega)}{\left(\Omega_{\mathrm{R}}-\Omega\right)^{3}} .
$$

Equations (4.6) and (4.7) are the solutions of the model. For the fast wave where $\Omega_{R}=+\sqrt{-U}$, we have $h^{\prime}\left(\Omega_{R}\right)<0$, and the denominator of (4.5) is positive; therefore $\Omega_{I}<0$. For the slow wave, on the other hand, $h^{\prime}\left(\Omega_{R}\right)>0$, and the denominator is negative; therefore, again, $\Omega_{I}<0$. Since the damping rate is $-\Omega_{I}$, we conclude that both waves are damped.

We have seen in Section $4 A$ that the coherent space-charge wave in a cold beam is stationary below transition energy. We see here that the frequency spread in the beam damps the waves out in the limit $t \rightarrow \infty$.

Equation (4.4) is the coherent condition: it gives the condition under which the beam particles can maintain coherence and thus support the coherent wave.

Suppose that the coherent condition is satisfied and that the corresponding coherent wave is excited, then Eq. (4.5) gives the Landau damping rate of this wave. We will see in the next section that Landau damping involves . one-way energy exchange (in contrast to the reactive energy exchange, which involves an exchange back and forth) between the beam particles and the EM fields, and that the numerator of $(4.5)$ is proportional to the rate of such a one-way energy exchange while the denominator is proportional to the total wave energy.

We close this section by a comment: Landau damping is not the same thing as damping by decoherence (or by "phase mixing"). Landau damping is damping of a coherent: wave; the condition for a coherent wave is in the present example given by.(4.4). One can have damping by decoherence without Landau damping. Let us illustrate this point by a simple example: Consider the case of a warm beam in an environment where $Z(\omega)=0$; the beam will then not be capable of carrying a coherent wave since the dispersion relation can never be satisfied. Suppose now that a collective motion of the beam particles is excited by an external impulse. For a suitable beam frequency spectnum, this motion will die away because of decoherence (or mathematicllly, by "Riemann-Lebesque lemma" of Fourier 
analysis). This damping, however, has. nothing to do with Landau damping, since no coherent wave is involved.

The results (4.4) and (4.5) will be rederived with an intuitive argument in the next section.

\section{LANDAU DAMPING AND RESONANT ENERGY EXCHANGE}

We consider again the model defined in the previous section for the longitudinal space-charge wave. Here we solve the model in a quite different but "more physical" manner; the central idea behind the present treatment is "energy balance."

Following Dawson, we divide the particle bearn into two parts: the main beam and the resonant particles, as illustrated in Fig. 2.
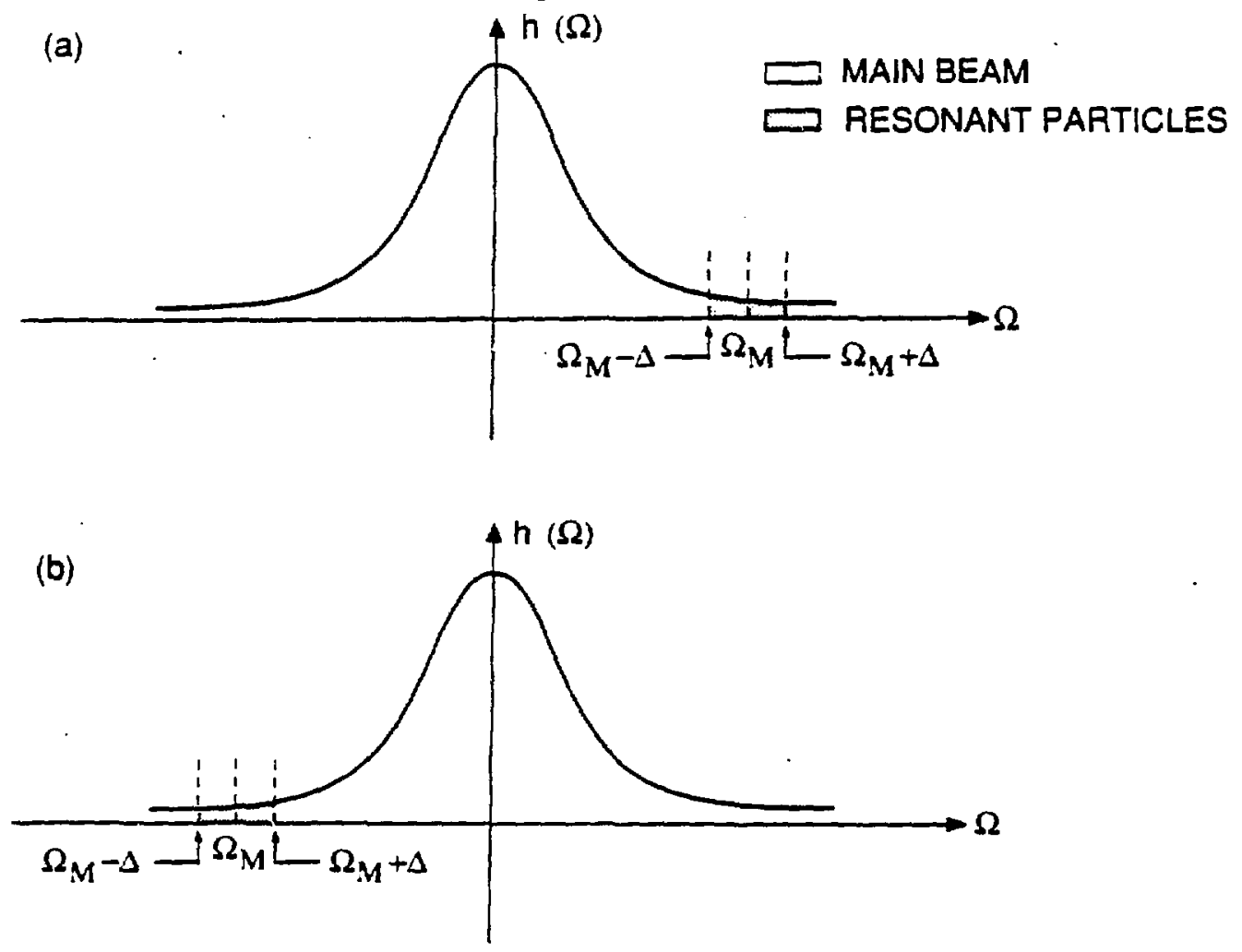

Fig. 2. Division of the beam into main beam and resonant particles;

(a) is for the fast wave and (b) is for the slow wave.

Suppose that a space-charge wave with coherent angular frequency $\omega_{M}=n \omega_{0}+\Omega_{M}$ is excited in the beam, the main beam will be considered to be the carrier of this wave. If the presence of the resonant particles is ignored, this wave will be stationary, since $V=0$, $U<0$, and the dispersion relation (5.19) below is not singular at $\Omega=\Omega_{M}$.

The resonance particles are those which rorate around the storage ring $\mathbf{w i t h}$ angular velocity lying in a very small interval between $\left(\omega_{M}-\Delta\right) / n$ and $\left(\omega_{M}+\Delta\right) / n$. $A$ 
resonant particle oscillates with a constant or nearly constant phase relationship to the space-charge wave. Therefore, it can interact strongly and have a one-way exchange of energy with the wave carried by the main beam. Landau damping will be demonstrated to be a consequence of this energy exchange. It will also be shown that the Landau damping rate calculated along this line of argument is the same as what we obtained in the last section.

Let us comment on the notation adopted in this section. We used $\alpha$ in Section 3 to denote the complex $n$-th Fourier component of the longitudinal polarization. In this section we have to deal both with such a quantiry and with the real part of it. We shall therefore denote the complex Fourier component by $\underline{\alpha}$, and the real part of it by $\alpha$. In other words,

$$
\dot{\alpha}(\theta, t)=\operatorname{Real}\left[\underline{\alpha} e^{i n \theta-i \omega t}\right]
$$

or

$$
\alpha(\theta, t)=\operatorname{Real}\left[\underline{\alpha}(t) e^{i n \theta}\right]
$$

Similar comments apply to other quantities like $E$ and $I$, etc.

A simple mathematical identity is very useful:

$$
\int_{0}^{2 \pi} d \theta A(\theta, t) B(\theta, t)=\pi \operatorname{Real}\left[\underline{A}(t) \underline{B}^{*}(t)\right] .
$$

\section{A. Kinetic Power Theorem}

When a coherent wave is excited, part of the wave energy will be in the particle beam as the beam-polarization energy and the rest of the energy will be in the EM fields in the beam chamber. Generally, there will be a reactive energy exchange between the beam particles and the EM fields. It is very important to understand the balance between these two parts of the energy.

We calculate here the beam polarization energy density and the polarization power: the technique used will be that of the kinetic power theorem (or "small-signal power theorem"). well-known in microwave engineering.

The equation of motion is

$$
\frac{d^{2}}{d t^{2}} \alpha(\theta, \varepsilon, t)=-\bar{\eta} E(\theta, t)
$$

with $\mathbf{d} / d t=\partial / \partial t+\omega_{0}(1-\eta \varepsilon) \partial / \partial \theta$. Let us denote

$$
v(\theta, \varepsilon, t)=\frac{d}{d t} \alpha ; \quad \dot{\alpha}=\frac{\partial \alpha}{\partial t} ; \quad \alpha^{\prime}=\frac{\partial \alpha}{\partial \theta} .
$$

Then, recalling from (2.7) that

$$
\mathrm{I}(\theta, \mathrm{t})=\frac{\mathrm{eN}}{2 \pi} \int \mathrm{d} \varepsilon \mathrm{g}(\varepsilon) \stackrel{\alpha}{\alpha}(\theta, \varepsilon, \mathrm{t}),
$$


and using this equation and (5.3), we can write

$$
\begin{array}{r}
I(\theta, t) E(\theta, t)=-\frac{N}{2 \pi \eta \bar{m}}\left[\frac{d}{d t} v(\theta, \varepsilon, t)\right] \int d \varepsilon^{\prime} g\left(\varepsilon^{\prime}\right) \dot{\alpha}\left(\theta, \varepsilon^{\prime}, t\right), \\
=-\frac{N}{2 \pi \bar{\eta}} \int d \varepsilon g(\varepsilon) \ddot{\alpha}(\theta, \varepsilon, t) \frac{d}{d t} v(\theta, \varepsilon, t) .
\end{array}
$$

The last equality follows from (5.4), since (5.4) is valid for any $\varepsilon$, and the left side of this equation is independent of $\varepsilon$. After a certain amount of algebra, (5.5) can be rewritten as

with

$$
I(\theta, t) E(\theta, t)=\frac{1}{R} \frac{\partial}{\partial \theta} P_{P}+\frac{\partial}{\partial t} u_{P}
$$

$$
P_{P}(\theta, \tau)=-\frac{N R}{2 \pi \eta} \int d \varepsilon g(\varepsilon) \omega_{0}(1-\eta \varepsilon) v \stackrel{\alpha}{\alpha}
$$

and

$$
u_{P}(\theta, t)=-\frac{N}{2 \pi \bar{\eta}} \int d \varepsilon g(\varepsilon)\left[\frac{1}{2} v^{2}-\omega_{0}\left(1-\eta \underline{v) v \alpha^{\prime}}\right] .\right.
$$

We have succeeded above in expressing $I(\theta, t) E(\theta, t)$ as a sum of a time derivative term and a spatial derivative term. Since $I(\theta, t) E(\theta, t)$ is the local rate of energy gain by the beam from the electric field $E(\theta, t)$, we can interprete $a_{\mathrm{p}}(\theta, t)$ as the polarization energ: density of the beam and $P_{P}(\theta, t)$ as the poiarization power.

If $\underline{I}(\theta, t)=1 \exp (\operatorname{in} \theta-i \omega t)$, then the induced longitudinal electric field is

$$
\underline{E}(\theta, t)=E e^{i n \theta-i \omega t}=-\frac{Z(\omega)}{2 \pi R} I e^{i n \theta-i \omega t} .
$$

Recalling that the impedance $Z$ is purely reactive, and using the definition (3.9), we have

$$
\dot{E}(\theta, t)=-\frac{2 \pi}{e^{2} N \bar{\eta}} U I(\theta, t)
$$

Therefore,

$$
I(\theta, t) E(\theta, t)=-\frac{e^{2} N \bar{\eta}}{2 \pi} \frac{1}{U} E(\theta, t) \stackrel{E}{E}(\theta, t)
$$

Now combine Eqs. (5.6) and (5.1!); the result is

$$
\frac{1}{R} \frac{\partial}{\partial \theta} P_{P}+\frac{\partial}{\partial t} u_{P}+\frac{e^{2} \vec{\eta}}{4 \pi} \frac{1}{U} \frac{\partial}{\partial t} E^{2}(\theta, t)=0 .
$$


This is the kinetic power theorem; it describes the relationship between the local polarization energy and the. electric energy density. The theorem implies that

$$
W_{\text {wave }} \equiv R \int_{0}^{2 \pi} d \theta\left[u_{p}(\theta, t)+\frac{e^{2} N \bar{\eta}}{4 \pi U} E^{2}(\theta, t)\right] \equiv W_{P}+W_{E B}
$$

is a conserved quantity. This is the total energy associated with the space-charge wave. $W_{\text {wave }}$ consists of two parts: the total polarization energy $W_{P}$ and the total electromagnetic wave energy $W_{E B}$.

\section{B. Energy of Space-Charge Wave}

We consider here only the main beam and ignore the presence of the resonant particles, and calculaie the energy $W_{\text {wave }}$ associated with the space-charge wave.

We start from the equation of motion of a particle in the main beam,

$$
\left[\frac{\partial}{\partial t}+\omega_{0}(1-\eta \varepsilon) \frac{\partial}{\partial \theta}\right]^{2} \alpha(\theta, \varepsilon, t)=-\bar{\eta} \overline{E_{M}}(\theta, t)
$$

where the longitudinal electric field is given by

$$
E_{M^{(\theta, t}}(\theta)=E_{M^{e}} e^{i n \theta-i \omega_{M} l}
$$

with $\omega_{M}$ the angular frequency of the space-charge wave. Let us take the solution of (5.14) to be

$$
\underline{\alpha}(\theta, \Omega, t)=\frac{e \bar{\eta}}{\left(\Omega_{M}-\Omega\right)^{2}} E_{M^{2}} e^{i n \theta-i \omega_{M^{2}}}
$$

with $\Omega_{M}=\omega_{M}-n \omega_{0}$. This, together with (2.7), implies

$$
\underline{I}_{M}(\theta, t)=-i \frac{e^{2} N \omega_{M} \bar{\eta}}{2 \pi} E_{M^{2}} e^{i n \theta-i \omega_{M} l} \int_{M} d \Omega \frac{h(\Omega)}{\left(\Omega_{M}-\Omega\right)^{2}},
$$

where $\int_{M}$ means integrating over the main beam; i.e.,

$$
\int_{M} d \Omega \equiv \int_{\Omega_{M}+\Delta}^{\infty} d \Omega+\int_{-\infty}^{\Omega_{M}-\Delta} d \Omega
$$


From (5.10),

$$
{\stackrel{E}{M^{\prime}}}(\theta, t)=-\frac{2 \pi}{e^{2} N \bar{\eta}} U \underline{I}_{M}(\theta, t)
$$

Substitution of (5.17) and (5.15) into (5.18) yields

$$
1+U \int_{M} d \Omega \frac{h(\Omega)}{\left(\Omega_{M}-\Omega\right)^{2}}=0 .
$$

Since $\Delta$ is by assumption a very small quantity, (5.19) is identical to (4.4), and we conclude

$$
\Omega_{\mathbf{M}}=\Omega_{\mathbf{R}} \text {. }
$$

Note that the integral in (5.19) is not singular, since the integration region $\mathbf{M}$ excludes the point $\Omega=\Omega_{\mathrm{M}}$ :

It is convenient to apply $(5.2)$ in calculating the total polarization energy $W_{p}$. Using $\underline{v}$ and $\underline{\alpha}^{\prime}$ calculated from $(5.16)$, we obtain from (5.8)

$W_{P} \equiv R \int_{0}^{2 \pi} d \theta u_{P}(\theta, t)=-\frac{1}{2} N e^{2} \bar{\eta} R\left|E_{M}\right|^{2} \int_{M} d \Omega h(\Omega)\left[\frac{1}{2} \frac{1}{\left(\Omega_{M}-\Omega\right)^{2}}+\frac{n \omega_{0}+\Omega}{\left(\Omega_{M}-\Omega\right)^{3}}\right]$

The second term of (5.13) is the total electromagnetic energy $W_{E B}$ associated with the space-charge wave. Noting, from (5.19), that

we obtain

$$
\frac{1}{U}=-\int_{M} d \Omega \frac{h(\Omega)}{\left(\Omega_{M}-\Omega\right)^{2}},
$$

$$
W_{\text {wave }}=W_{P}+W_{E B}=-\frac{1}{2} N e^{2} \bar{\eta} R\left|E_{M}\right|^{2} \omega_{M} \int_{M} d \Omega \frac{h(\Omega)}{\left(\Omega_{M}-\Omega\right)^{3}} .
$$

Note that $W_{\text {wave }}$ is positive for the fast wave and negative for the slow wave, since $\eta<0$.

It is instructive to write $(5.21)$ in another form. First, it is demonstrated in the Appendix that

$$
w_{E \perp}-w_{B \perp}=\left(e^{2} N \bar{\eta} R / 4 U\right)\left|E_{M}\right|^{2} \text {, }
$$

where the left side is the total transverse reactive electromagnetic energy associated with the wave. Second, if we define the dielectric function of the main beam, 


$$
D_{\left.M^{(}\right)}(\omega) 1+U \int_{M} d \Omega \frac{h(\Omega)}{\left(\omega-n \omega_{0}-\Omega\right)^{2}},
$$

then, using (5.19), we have

$$
\left.\frac{\partial}{\partial \omega}\left[\omega D_{M}(\omega)\right]\right|_{\omega=\omega_{M}}=-2 U \omega_{M} \int_{M} d \Omega \frac{h(\Omega)}{\left(\Omega_{M}-\Omega\right)^{3}} .
$$

Therefore, the wave energy becomes

$$
w_{\text {wave }}=\left.\left(W_{E \perp}-w_{B \perp}\right) \frac{\partial}{\partial \omega}\left[\omega D_{M}(\omega)\right]\right|_{\omega=\omega_{M}} .
$$

Not surprisingly, the reactive electromagnetic energy plays a crucial role in the spacecharge wave.

\section{C. Resonant Energy Exchange and Damping Rate}

We now calculate the rate of energy exchange between the resonant particles and the space-charge wave carried by the main particles.

Again, start from the equation of motion for the resonant particles,

$$
\frac{d^{2}}{d^{2}} \alpha(\theta, \varepsilon, t)=-e \bar{\eta} E_{M}(\theta, t) \text {. }
$$

This equation is of the same form as (5.14), but the range of $\varepsilon$ is different. Equation (5.16) is still a solution of (5.23). However, $\alpha$ as given by this equation is very large, since $\Omega$ for the resonant particle is very close to $\Omega$; this will invalidate the linear approximation. Let us therefore treat (5.23) as an initial value problem. Also, let us assume for simplicity that $\alpha$ and its derivative vanish at $t=0$. Then,

$\underline{\alpha}(\theta, \Omega, t)=\frac{e \bar{\eta}}{\left(\Omega_{M}-\Omega\right)^{2}} E_{M} e^{i n \theta}\left[e^{-i \omega_{M}{ }^{l}}-e^{-i\left(n \omega_{0}+\Omega\right) t}+i t\left(\Omega_{M}-\Omega\right) e^{-i\left(n \omega_{0}+\Omega\right) t}\right]$.

The resonant current is

$$
\underline{I}_{\text {res }}(\theta, \mathrm{t})=\frac{e N}{2 \pi} \int_{\text {res }} \mathrm{d} \Omega \mathrm{h}(\Omega) \underline{\dot{\alpha}}(\theta, \Omega, \mathrm{t}),
$$

and the rate of resonant energy absorption is, using (5.2),

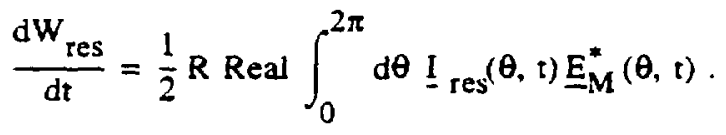


From the last three equations,

$$
\begin{gathered}
\frac{d W_{\text {res }}}{d t}=\frac{1}{2} e^{2} N \bar{\eta} R\left|E_{M}\right|^{2} \int_{\text {res }} d \Omega h(\Omega)\left[-\omega_{M} \frac{\sin \left(\Omega_{M}-\Omega\right) t}{\left(\Omega_{M}-\Omega\right)^{2}}-\right. \\
\left.t \cos \left(\Omega_{M}-\Omega\right) t+\frac{\omega_{M}}{\Omega_{M}-\Omega} t \cos \left(\Omega_{M}-\Omega\right) t\right]
\end{gathered}
$$

We want to evaluate $(5.27)$ in the limit $t \rightarrow \infty$. In terms of an integration variable

this equation. becomes

$$
\mathbf{x}=\left(\Omega-\Omega_{M}\right) t
$$

$$
\begin{aligned}
W_{r e s}(t)= & \frac{1}{2} e^{2} N \bar{\eta} R\left|E_{M}\right|^{2} \cdot \frac{1}{t} \int_{-\Delta t}^{\Delta t} d x h\left(\Omega_{M}+\frac{x}{t}\right) \\
& \times\left[\omega_{M} t^{2} \frac{\sin x}{x^{2}}+t \cos x-\omega_{M^{t^{2}}} \frac{1}{x} \cos x\right]
\end{aligned}
$$

We observe that the integrand is well behaved at $x=0$; therefore, the integral can be replaced by the principal integral at $x=0$. The integrand consists of three terms, but the principal integral of a sum is a sum of principal integrals, with each principal integral well behaved at $x=0$.

The last principal integral does sout contribute in the limit $t \rightarrow \infty$, since

$$
f_{-\Delta t}^{\Delta t} \mathrm{dxh}\left(\Omega_{M}+\frac{x}{t}\right) \frac{1}{x} \cos \mathrm{x} \equiv f_{-\Delta t}^{\Delta t} \mathrm{dxh}\left(\Omega_{M}\right) \frac{1}{x} \cos x=0 \text { ， }
$$

from symmetry.

To evaluate the second integral, note that we can let $\mathrm{t} \rightarrow \infty$ in steps since the integral is well behaved at $\mathrm{x}= \pm \infty$. Set $\mathrm{t}=2 \pi \ell / \Delta$ with integer $\ell$, and let $\ell \rightarrow \infty$. Again, $h\left(\Omega_{M}+x / t\right) \equiv h\left(\Omega_{M}\right)$, and the integral vanishes since cos $x$ integrated over a period is zero.

We are left with the first integral. To evaluate this, expand

$$
h\left(\Omega_{M}+\frac{x}{t}\right) \equiv h\left(\Omega_{M}\right)+h^{\prime}\left(\Omega_{M}\right) \frac{x}{t}+\cdots
$$

The first term of this expansion does not contribute to the integral, from symmetry; theiefore we are left with

$$
\int_{-\Delta t}^{\Delta t} d x h\left(\Omega_{M}+\frac{x}{t}\right) \frac{\sin x}{x^{2}} \underset{t \rightarrow \infty}{\rightarrow} \frac{1}{t} h^{\prime}\left(\Omega_{M}\right) \int_{-\infty}^{\infty} d x \frac{\sin x}{x}=\frac{\pi}{t} h^{\prime}\left(\Omega_{M}\right) .
$$


We conclude that

$$
\dot{w}_{\text {res }}=R \int_{0}^{2 \pi} d \theta I_{\text {res }}(\theta, t) E(\theta, t)=\frac{\pi}{2} e^{2} \bar{N} \bar{\eta} R\left|E_{M}\right|^{2} \omega_{M} h^{\prime}\left(\Omega_{M}\right)
$$

in the limit $t \rightarrow \infty$.

Since $h^{\prime}\left(\Omega_{M}\right) \geqslant 0$ for $\Omega_{M} \lessgtr 0$, and $\eta<0$, the resonant particles absorb energy from the fields for fast waves, and they give out energy for slow waves.

If we set

$$
\mathbf{w}_{\text {res }}=-\mathbf{w}_{\text {wave }}
$$

and define the damping rate of the wave energy by

$$
\delta=-\dot{w}_{\text {wave }} / w_{\text {wave }},
$$

then, combining the above equations and (5.21), we find

$$
\delta=-\pi h^{\prime}\left(\Omega_{M}\right) / \int_{M} \mathrm{~d} \Omega \frac{\mathrm{h}(\Omega)}{\left(\Omega_{M}-\Omega\right)^{3}} .
$$

This agrees with what we found in Section 4 by solving the dispersion relation. Comparing (4.7) with (5.32), we have.

$$
\delta=-2 \Omega_{\mathrm{I}} \text {. }
$$

The factor -2 comes from the fact that $\delta$ is the damping rate of the energy and $\Omega_{I}$ is the growth rate of the amplitude.

This completes the demonstration that the Landau damping of the space-charge wave is a consequence of the one-way energy exchange between the wave and the resonant particles in the beam.

\section{APPENDIX: LONGITUDINAL SPACE-CHARGE IMPEDANCE}

We first review the well-known properties of EM fields induced by a longitudinally perturbed uniform coasting beam with radius a moving in the center of a beam chamber with a perfectly conducting chamber wall and with radius $b$. Later on, we will assume $\mathrm{a} / \mathrm{b} \ll<\mathrm{I}$, so that the longitudinal electric field $\mathrm{E}$ can be assumed to be a constant across the beam; the ultimate goal is to relate this constant $E$ to the reactive $E M$ energy in the ring.

The quantities $E, \vec{B}, J$, and $\rho$ are all assumed to be proportional to $\exp (i n \theta-i \omega t)$. Under the assumption that $b^{2} k^{2} / \gamma_{w}^{2} \ll 1$, the longitudinal electric field $E$ is given by 


$$
E=-i \frac{2 k}{\gamma_{w}^{2} \beta_{w} c} I_{a v} \begin{cases}\ln \frac{b}{a}+\frac{1}{2} \frac{a^{2}-r^{2}}{a^{2}}, & 0<r<a, \\ -\ln \frac{r}{b}, & a<r<b,\end{cases}
$$

where $k=n / R, R=$ ring radius, $\beta_{w} c=\omega / k, \gamma_{w}=\left(1-\beta_{w}^{2}\right)^{-1 / 2}$, and $I_{a v}=e N \omega_{0} / 2 \pi$. Also,

$$
E_{r}=\frac{2}{\beta_{w} c} I_{a v} \begin{cases}r / a^{2}, & r<a, \\ 1 / r, & a<r<b,\end{cases}
$$

and $B_{\varphi}=\beta_{w} E_{t}$. The other components of the EM fields vanish.

Note that this approximate solution satisfies the the following equations:

$$
\begin{array}{ll}
\vec{\nabla}_{\perp} \cdot \vec{E}_{\perp}=4 \pi \rho, & \vec{\nabla} \times \vec{E}=-\frac{1}{c} \vec{B}, \\
\vec{\nabla} \cdot \vec{B}=0, & \vec{\nabla} \times \vec{B}=\frac{4 \pi}{c} \vec{J}+\frac{1}{c} \vec{E}_{\perp},
\end{array}
$$

where $\vec{J}$ is along the longirudinal direction, $\vec{J}=J \hat{z}, J=I_{a v} / \pi a^{2}$, and $\vec{E}_{\perp}$ is the transverse component of the electric field.

The complex Poynting's theoren corresponding to the modified Maxwell's equations above is

$$
\frac{1}{2} J^{*} E+\frac{c}{8 \pi} \vec{\nabla} \cdot\left(\vec{E} \times \vec{B}^{*}\right)+i \omega \frac{1}{8 \pi}\left(\left|\vec{E}_{\perp}\right|^{2}-\left|\vec{B}_{\perp}\right|^{2}\right)=0
$$

Note that only the transverse reactive EM energy density contributes to the above theorem. Defining

$$
W_{E \perp} \equiv \frac{1}{16 \pi} \int_{\text {ring }} d^{3} x\left|\vec{E}_{\perp}\right|^{2}, \quad W_{B \perp} \equiv \frac{1}{16 \pi} \int_{\text {ring }} d^{3} x\left|\vec{B}_{\perp}\right|^{2}
$$

and integrating the above theorem over the storage ring. we obtain

$$
\frac{1}{2} \int_{\text {beam }} d^{3} x J^{*} E+2 i \omega\left(W_{E \perp}-W_{B \perp}\right)=0
$$


If the definition for the impedance $Z(\omega)$,

$$
\int_{\text {beam }} d^{3} x J^{*} E=I_{a v}^{2} Z(\omega),
$$

and the explicit expressions for EM fields given above are used, (A.3) becomes

$$
\begin{aligned}
\frac{1}{2} I_{a \psi}^{2} Z & =2 i \omega\left(W_{E \perp}-W_{B \perp}\right) \\
& =i 2 \pi I_{a v}^{2} \frac{n}{\gamma_{w}^{2} \beta_{w} c}\left(\ln \frac{b}{a}+\frac{1}{4}\right)
\end{aligned}
$$

From now on we assume further that $b / a \gg 1$, so that (A.1) can be approximated by

$$
E=-i\left(2 k / \gamma_{w}^{2} \beta_{w} c\right) i_{a v} \ln b / a, \quad r<a .
$$

$E$ is a constant across the beam within this approximation.

To relate $E$ to the reactive energy, we observe from (A.4) and the constancy of $E$ that

$$
E=-\frac{1}{2 \pi R} I_{a v} Z(\omega) \text {. }
$$

A combination of this equation with (A.5) gives

$$
W_{E \perp}-W_{B \perp}=\pi^{2} R^{2}|E|^{2} / i \omega Z^{*}
$$

Since $\mathbf{Z}$ is purely reactive, we can write this expression, using the definition (3.9) in Section 3 , as

$$
W_{E \perp}-w_{B \perp}=\left(e^{2} \bar{\eta} N R / 4 U\right)|E|^{2} .
$$

This relation between the longitudinal electric field density and the total transverse reactive energy is used in Section 5 to express the space-charge wave energy in terms of the reactive energy.

ACKNOWLED(jEMENT I thank John Galayda, Mel Month, and Jim Murphy for discussions. This work was supported by the U.S. Department of Energy under contract DE-ACO2-76CH00016.

\section{REFERENCES}

1. L. D. Landau, J. Phys. USSR 10, 25 (1946).

2. S. Ichimanu, Plasma Physics (W. A. Benjamin. Inc., Reading, MA, 1973).

3. J. J. Bisognano, Proc. of 1986 Joint U.S.-CERN Accelerator School, p. 247 (SpringerVerlag, Berlin-Heidelberg, 1988). 
4. S. Chattopadhyay, 1983 U.S. School on Particle Accelerators, AIP Proc. 127, p. 469

5. A. Hoffman and B. Zotter, IEEE Trans. Nucl. Sci. NS-24 (3) 1487 (1977).

6. J. M. Wang. 1985 U.S. School on Particle Accelerators, AIP Proc. 153, p. 697.

7. J. Dawson, Phys. Fluids \&, 869 (1961).

8. H. G. Hereward, CERN Repor 65-20 (1965).

9. L. J. Chu, presented at IRE-PGED Conf., Durham, NH, 1951; quoted in Refs. 10 and 11 .

10. R. E. Collin, Foundations for Microwave Engineering, Section 9.5 (McGraw-Hill, New York, 1966).

11. W. H. Louisell, Coupled Mode and Parametric Electronics (Wiley, New York, 1960).

12. C. E. Nielson, A. M. Sessler, and K. R. Symon, Proc. In. Conf. on Accelerators, p. 239, (CERN, Geneva, 1959).

13. V. K. Neil and A. M Sessler, Rev. Sci. Instrum. 36, 429 (1965). 